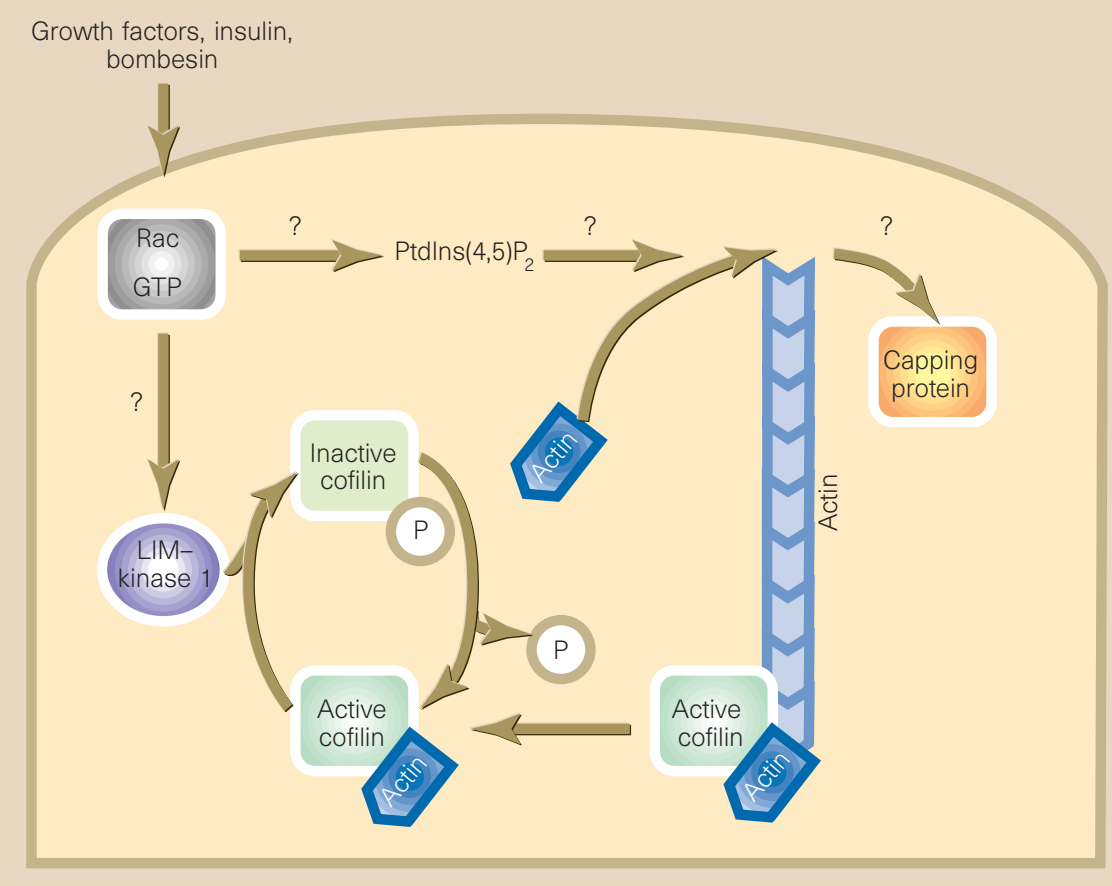

Figure 1 Model for Rac-induced changes in actin dynamics to form a lamellipodium, based on the findings of Arber et al. ${ }^{3}$ and Yang et al. ${ }^{4}$. When Rac is stimulated by growth factors, it activates LIMkinase 1 (LIMK-1) which, in turn, phosphorylates cofilin. This may inhibit localized disassembly of actin filaments and help to release cofilin from the actin monomers. Rac-mediated stimulation of actin polymerization may occur through uncapping or increased nucleation of actin filaments. Both of these mechanisms may occur through production of phosphatidylinositol-4,5-bisphosphate $\left(\operatorname{PtdIns}(4,5) \mathrm{P}_{2}\right)$ by phosphatidylinositol phosphate 5-kinase. The intermediates that activate phosphatidylinositol phosphate 5-kinase and LIMK-1, and the factor that stimulates dephosphorylation of cofilin, are unknown.

tion and dephosphorylation would allow both cofilin and actin to be recycled for further rounds of depolymerization and polymerization, respectively. Clearly, further work is needed to sort out the role of cofilin phosphorylation in actin dynamics, and to clarify the temporal and spatial regulation of actin depolymerization in cells.

Another exciting aspect of this work comes from studies on patients with Williams syndrome, who have only one copy of the LIMK-1 gene. Given that LIMK-1 regulates the turnover of actin filaments, why should people with Williams syndrome have defects in visuo-spatial cognition, as opposed to other processes that require actin dynamics? Perhaps neurons require high levels of LIMK-1 to finely regulate the turnover of actin filaments during axonal guidance. The PC12 neuronal cells containing dominant-negative LIMK-1 studied by Arber et al. may provide a clue as to what neurons with decreased levels of LIMK-1 look like. Although neuron outgrowth still occurred, the neurites contained dramatically fewer filopodia - finger-like projections that are thought to sense in which direction axons should grow. If this is true, a decrease in LIMK- 1 could account for the abnormally clustered and aligned neurons seen in the brains of patients with Williams syndrome ${ }^{12}$.
To show that a decrease in LIMK-1 leads to abnormal neuronal wiring, we need to study people who lack a copy of the LIMK-1 gene (and not contiguous genes), as well as mouse knockout models. Future work will also need to address whether the visuo-spatial cognitive defect in people with Williams syndrome results exclusively from phosphorylation of cofilin by LIMK-1, or whether other substrates for LIMK-1 exist.

Jody Rosenblatt is in the Department of

Biochemistry, University of California at San

Francisco, 513 Parnassus Avenue, San Francisco,

California 94143, USA.

e-mail: jody@cgl.ucsf.edu

Timothy J. Mitchison is in the Department of Cell

Biology, Harvard Medical School, Room C517,

240 Longwood Avenue, Boston, Massachusetts

02115, USA.

e-mail:Timothy_Mitchison@hms.harvard.edu

1. Frangiskakis, J. M. et al. Cell 86, 59-69 (1996).

2. Okano, I. et al. J. Biol. Chem. 270, 31321-31330 (1995).

3. Arber, S. et al. Nature 393, 805-809 (1998).

4. Yang, N. et al. Nature 393, 809-812 (1998).

5. Theriot, J. A. \& Mitchison, T. J. Nature 352, 126-131 (1991).

6. Abe, H., Obinata, T., Minamide, L. S. \& Bamburg, J. R. J. Cell Biol. 132, 871-885 (1996).

7. Lappalainen, P. \& Drubin, D. G. Nature 388, 78-82 (1997).

8. Theriot, J. A. J. Cell Biol. 136, 1165-1168 (1997).

9. Moon, A. \& Drubin, D. G. Mol. Biol. Cell 6, 1423-1431 (1995).

10. Ridley, A. J., Paterson, H. F., Johnston, C. L., Diekmann, D. \& Hall, A. Cell 70, 401-410 (1992).

11. Hartwig, J. H. et al. Cell 82, 643-653 (1995).

12. Galaburda, A. M., Wang, P. P., Bellugi, U. \& Rossen, M.

Neuroreport 5, 753-757 (1994).
Daedalus

\section{Muffled furnace}

Noise is one of the major nuisances of modern life. Yet the usual sound-absorbing materials are purely passive, and can never damp it out completely. Daedalus now proposes an active absorber, inspired by the observation in a chemical textbook that the carbon monoxide flame "gives a curious impression of silence". Carbon monoxide, he notes, burns with a reduction in the number of gas molecules. If, like most gas reactions, the burning speeds up with pressure, then a sonic pressure-peak will deplete the gas of molecules at an enhanced rate, and damp itself out. Conversely, when the pressure is low, the depletion slows down. So the flame absorbs the sound. Furthermore, certain flames are extremely sensitive to sound. They were used as acoustic detectors in pre-microphone days. Even a weak sound changed their combustion regime very visibly.

So Daedalus is inventing quiet flame technology. He is devising nozzles and flame-surrounds that optimize this sounddamping effect. He hopes to perfect a gas burner whose nonlinear reaction regime overreacts to sound, and thus absorbs it perfectly. A street lamp that absorbed traffic noise would be welcome on busy roads; victims of aircraft noise or popcrazed neighbours would love a gas fire that gave out quiet as well as heat.

Even so, nobody would want to keep a fire burning on a hot day, merely to keep the noise down. So Daedalus is taking the argument further. When iron rusts, for example, gas molecules are completely absorbed into a solid. DREADCO's chemists are now studying the oxidation of iron alloys, as well as yellow phosphorus, aluminium amalgam and even lithium (which absorbs nitrogen as well). They are seeking pressure-sensitive reaction regimes with strong nonlinearity, or even a pressure threshold. Their goal is a surface that rusts or tarnishes with total absorption of sound. Ideally it should change colour during the reaction. When fully reacted, it could be regenerated, perhaps by reduction with hydrogen.

This novel decor will be very expensive at first, and will be aimed at acoustics laboratories and recording studios. Gradually it should spread to the more opulent homes, offices and public buildings. A personal version in earmuff form would be widely welcomed; not only for the quiet in which it wrapped the wearer, but for the pleasing warmth released by its slow sonic oxidation. David Jones 